\title{
Impact of luteal phase hysteroscopy and concurrent endometrial biopsy on subsequent IVF cycle outcome
}

\author{
Tarek Shokeir
}

Received: 29 September 2014/Accepted: 5 December 2014/Published online: 23 December 2014

(C) Springer-Verlag Berlin Heidelberg 2014

Dear Editor,

We have read the paper published by Kumbak et al. [1] with great interest. The clinical data reported by the authors support the proposed theoretical mechanism by which luteal phase endometrial injury-induced inflammation improves uterine receptivity, implantation and pregnancy outcome in the subsequent ET cycle. However, these results contradict others' published as systematic reviews and meta-analyses $[2,3]$. It is expected that a suggested favourable influence of luteal phase local endometrial injury would not be sustained and could be lessened by shedding of the affected target endometrium in the subsequent menses before ET.
Conflict of interest We declare no conflict of interest.

\section{References}

1. Kumbak B, Sahin L, Ozkan S, Atilgan R (2014) Impact of luteal phase hysteroscopy and concurrent endometrial biopsy on subsequent IVF cycle outcome. Arch Gynecol Obstet 290(2):369-374

2. Karimzade MA, Oskouian H, Ahmadi S, Oskouian L (2010) Local injury to the endometrium on the day of oocyte retrieval has a negative impact on implantation in assisted reproductive cycles: a randomized controlled trial. Arch Gynecol Obstet 281(3):499-503

3. Simón C, Bellver J (2014) Scratching beneath 'The Scratching Case': systematic reviews and meta-analyses, the back door for evidence-based medicine. Hum Reprod 29(8):1618-1621
This comment refers to the article available at doi:10.1007/s00404014-3211-y and an author's reply to this comment is available at doi:10.1007/s00404-014-3584-y.

T. Shokeir $(\square)$

Department of Obstetrics and Gynecology, Mansoura University

Hospital, Mansoura Faculty of Medicine, Mansoura, Egypt

e-mail: tarek.shokeir@gmail.com 